\title{
A Novel Method of Thrombus Preparation for Use in a Swine Model for Evaluation of Thrombectomy Devices
}

I. Kan

SUMMARY: We report the development of a novel technique of thrombus preparation for use in a

I. Yuki

Y. Murayama swine model for evaluation of thrombectomy devices. The experimental thrombus prepared using plain sedimentation showed mechanical stability due to its solid fibrin-rich component. The thrombus also showed histologic similarity to the typical thromboemboli recovered from patients with stroke in

F.A. Viñuela the course of therapeutic thrombectomy. This new technique may be beneficial for preclinical evaluR.H. Kim ation of thrombectomy devices.

E ndovascular thrombectomy is an effective method for the treatment of acute ischemic stroke. ${ }^{1}$ A swine model described by Gralla et $\mathrm{al}^{2}$ is currently one of the most commonly used animal models for evaluation of mechanical thrombectomy devices; however, several technical limitations remain. First, the typical experimental thrombus used in the model represents a homogeneous erythrocyte-rich clot, which is mechanically unstable and tends to fragment during the procedure. Second, its histologic characteristics are not necessarily consistent with those of thromboemboli recovered from patients with stroke. Recent studies showed that most of the thrombi recovered from patients with acute stroke are composed of multiply layered patterns, including a fibrin-rich layer, a cellular component, and an erythrocyte-rich layer. ${ }^{3}$

We describe a novel technique of thrombus preparation for use in a swine model for evaluation of thrombectomy devices. The new experimental thrombus appeared to be mechanically stable and histologically similar to typical thromboemboli recovered from patients with stroke.

\section{Materials and Methods}

\section{Animal Care}

All animal experiments were conducted in accordance with policies set by the Chancellor's Animal Research Committee of the University

\section{Received July 14, 2009; accepted after revision November 6 .}

From the Department of Radiological Sciences (I.K., I.Y., Y.M., F.A.V., R.H.K., F.V.), Division of Interventional Neuroradiology, Section of Neuropathology, and Departments of Pathology and Laboratory Medicine and Neurology (H.V.V), David Geffen School of Medicine, University of California, Los Angeles, Los Angeles, California.

H.V.V. is supported in part by the Daljit S. and Elaine Sarkaria Chair in Diagnostic Medicine and SPOTRIAS grant 1P50NS044378.

Paper previously presented in part at: International Stroke Conference, February 17-20, 2009; San Diego, California; and Annual Meeting of American Society of Neuroradiology May 16-21, 2009; Vancouver, British Columbia, Canada.

Please address correspondence to Issei Kan, MD, Division of Interventional Neuroradiology, Department of Radiological Sciences, UCLA Medical Center and David Geffen School of Medicine at UCLA, 10833 Le Conte Ave, Los Angeles, CA 90095-1721; e-mail: ikan@ mednet.ucla.edu

\section{Indicates open access to non-subscribers at www.ajnr.org}

DOI 10.3174/ajnr.A1991 of California, Los Angeles. A total of 2 Yorkshire swine were used in this study.

\section{Preparation of New Experimental Thrombus}

Twenty milliliters of whole blood was obtained from a 7F sheath, which was placed in the right femoral artery of a swine. The content was then mixed with $2 \mathrm{~g}$ of barium sulfate powder to obtain radiopacity. To minimize the barium's anticoagulant effect, which is generally explained by absorption of the K-dependent coagulation factor, ${ }^{4}$ we paid special attention to the rotational motion when the content was mixed. The syringe was slowly rotated for $10-30$ seconds so that the blood component was partially mixed with the barium layer formed at the bottom. Vigorous mixing was avoided. The mixture in the syringe was incubated at room temperature for 120 minutes until it showed a multiply-layered structure of blood constituent and barium. After the sedimentation, a solid component was separated from the serum component. The solid component showed 2 layers, a fibrin-rich layer and an erythrocyte-rich layer.

Using a number 11 surgical scalpel, we carefully resected a small piece of clot, measuring approximately $5 \mathrm{~mm}$ in diameter and $20 \mathrm{~mm}$ in length, from the aforementioned solid component with both fibrin-rich and erythrocyte-rich layers. Each prepared thrombus was then inserted into a silicone tube (MX 451 FL; Medex, Dubin, Ohio) with saline and prepared for injection.

\section{Preparation of Conventional Experimental Thrombus (Thrombin-Induced Thrombus)}

As a control, a conventional experimental thrombus (thrombin-induced thrombus) was also prepared. The detailed preparation process is described elsewhere. ${ }^{2}$ Briefly, $10 \mathrm{~mL}$ of swine whole blood was mixed with $25 \mathrm{IU}$ of bovine thrombin (Dade Behring, Newark, Delaware). One gram of barium sulfate was mixed with the blood component for 10 seconds, and the mixture was kept in a silicone tube for 60 minutes at room temperature. After the incubation period, the created clot was removed from the silicone tube and cut to a length of $20 \mathrm{~mm}$.

\section{Assessment of Mechanical Properties (Manual Elongation Test)}

Ten samples were selected from each group, and a qualitative assessment of manual elongation was performed by using forceps. 

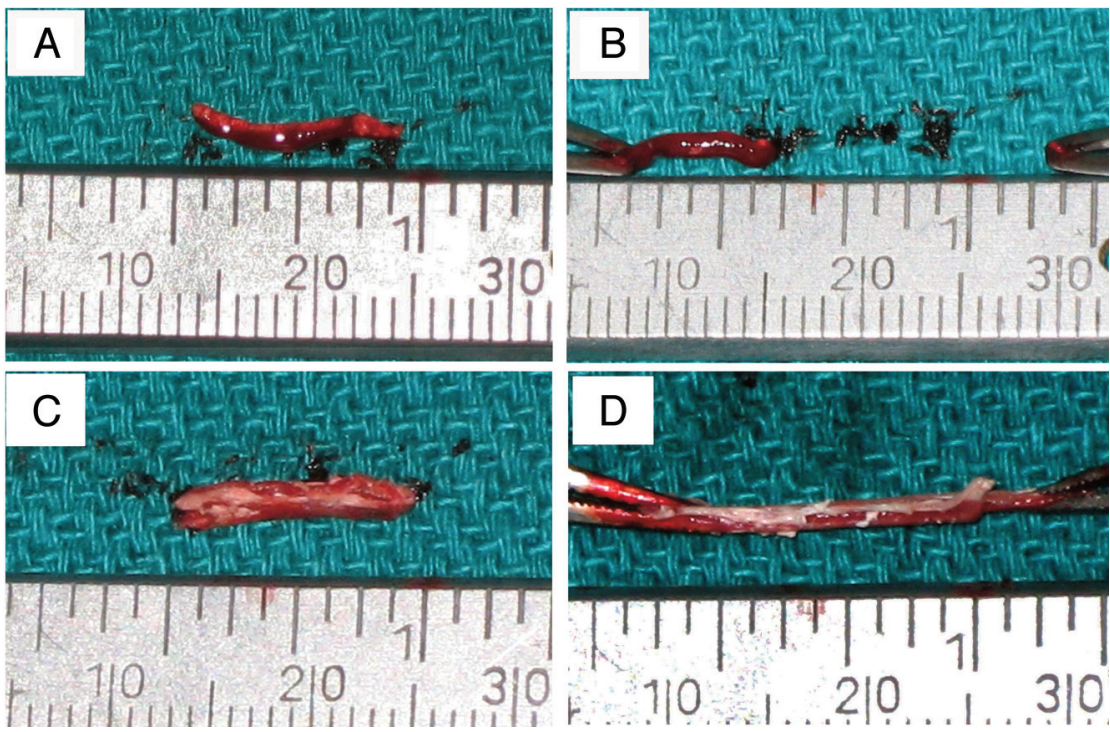

Fig 1. Photographs showing a manual elongation test of a thrombin-induced thrombus $(A$ and $B$ ) and a new experimental thrombus $(C$ and $D)$. Note that most of the thrombin-induced thrombus was torn before it attained a doubling of its length $(A$ and $B)$, whereas the new experimental thrombi were more elastic and solid and most of them tolerated stretching to 3 times the length of the original size $(C$ and $D)$.


Fig 2. Photomicrographs of 3 different types of thrombus. $A$, Thrombin-induced thrombus was replete with erythrocytes and lacked a layered structure. $B$, Most thromboemboli recovered from patients with stroke showed a layered structure, including fibrin-rich $(F)$ and erythrocyte-rich $(E)$ layers. $C$, New experimental thrombus presented a layered pattern similar to that observed in the thromboemboli recovered from patients with stroke $(B)$. C indicates a cellular component; $\mathrm{E}$, an erythrocyte-rich component; $\mathrm{F}$, a fibrin-rich component (hematoxylin-eosin, original magnification $\times 40$ ).

\section{Thromboembolization of External Carotid Arteries with the New Experimental Thrombus}

With the swine under general anesthesia, we advanced a $6 \mathrm{~F}$ guiding catheter (Envoy; Cordis, Miami Lakes, Florida) through the femoral sheath and placed it in the right lingual artery. After connecting the silicone tube containing a prepared clot to the guiding catheter, we injected $2 \mathrm{~mL}$ of saline into the tube to push the clot into the guiding catheter. Next, $3 \mathrm{~mL}$ of saline was injected into the guiding catheter under fluoroscopy to inject the clot into the target artery. The same procedure was repeated in the ipsilateral internal maxillary artery and the ascending pharyngeal artery.

\section{Angiographic Evaluation}

Postprocedural angiography was performed after the thromboembolization to evaluate the following: 1) the radiopacity of each injected thrombus, 2) fragmentation of injected thrombus, 3) total/partial occlusion of each target artery, and 4) induced vasospasm.

\section{Histologic Analysis}

We analyzed 10 samples of the following types of thrombus: 1) new experimental thrombi, 2) thrombin-induced thrombi, and 3) thromboemboli retrieved from patients with stroke. Each sample was fixed in $10 \%$ buffered formalin. Samples from the patients were thromboemboli retrieved from the middle cerebral arteries of patients with acute ischemic stroke by using the Merci Retrieval System (Concentric Medical, Mountain View, California). The specimens were embedded in paraffin, cut at $5-\mu \mathrm{m}$ thickness, and processed with hematoxylin-eosin staining. Histologic examination was performed by a neuropathologist (H.V.V).

\section{Results}

\section{Macroscopic Findings of Experimental Thrombus}

The control samples, the thrombin-induced thrombi, showed a homogeneous, dark-red, and gel-like appearance (Fig 1A), 

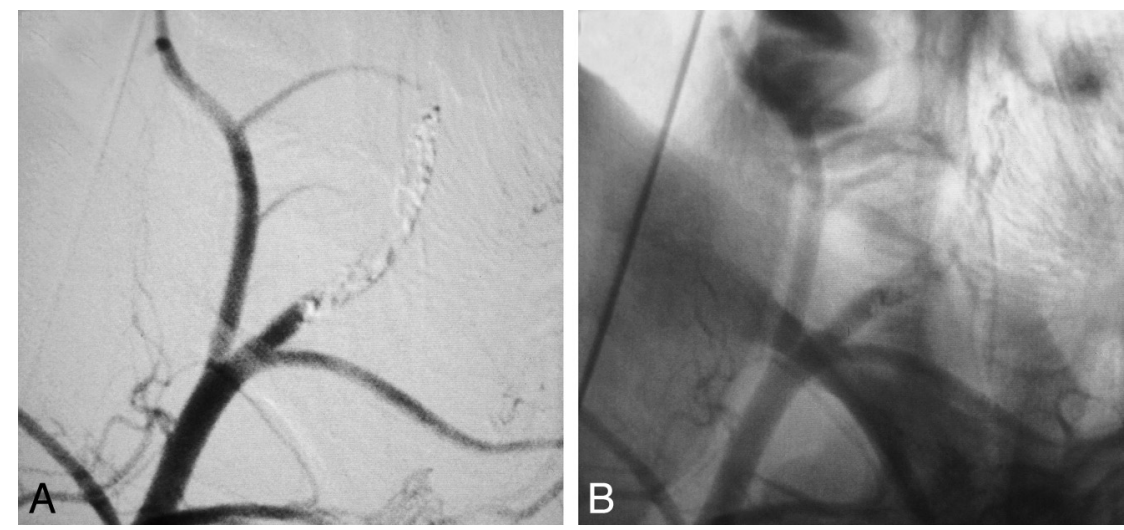

Fig 3. Representative angiograms after deployment of the new experimental thrombus (lateral view, right common carotid artery injection). $A$, Complete occlusion of an internal maxillary artery was confirmed with a digital subtraction image. $B$, Nonsubtracted image showed sufficient radiopacity of the injected thrombus.

whereas our new experimental thrombi presented a multiplylayered appearance composed of both white and reddish layers (Fig 1C). The manual elongation test showed that most thrombin-induced thrombi ( 9 of 10) were torn before they reached double their initial length, whereas most new experimental thrombi ( 8 of 10) were more elastic and solid and tolerated stretching to a length of 3 times that of the original size (Fig 1).

\section{Histologic Results}

Histologically, every sample from the thrombin-induced thrombus was replete with erythrocytes and lacked a fibrinrich and cellular component (Fig 2A). In contrast, most thromboemboli recovered from patients with stroke presented a layered pattern including both fibrin-rich and erythrocyte-rich layers (Fig $2 B$ ). A cellular component, which was mainly composed of linear neutrophils, monocytes, and platelets was also observed. A similar layered pattern was observed in the samples from the new experimental thrombi (Fig 2C).

\section{Thromboembolization and Angiographic Results}

Complete occlusion of 4 internal maxillary arteries, 4 ascending pharyngeal arteries, and 4 lingual arteries was successfully achieved in 2 swine. Sufficient radiopacity of each injected thrombus was confirmed (Fig 3). Neither fragmentation of injected thrombus nor associated vasospasm was seen during the procedure.

\section{Discussion}

The ideal stroke model for evaluation of a mechanical thrombectomy device is an animal model that reproduces the mechanical interaction between a thromboembolus and the device used in a given patient with stroke. The purpose of this study was to reproduce an experimental thrombus that is mechanically stable and histologically similar to the thromboemboli recovered from patients with stroke.

In the study, gentle rotation of a syringe containing whole blood and barium sulfate was performed followed by relatively longer plain sedimentation of the mixture. It is possible that this maneuver allowed us to create a solid thrombus with sufficient radiopacity without using exogenous thrombin, despite the mild anticoagulant effect of the barium. In addition, the maneuver appeared to be associated with the formation of a unique layered pattern of the created thrombus. This study does not include data about how the new experimental thrombus behaves when subjected to the forces resulting from the application of thrombectomy devices. Additional experiments using thrombectomy devices are required to evaluate how different compositions of thrombi influence the performance of a thrombectomy device.

The histologic characteristics of thromboembolus vary from patient to patient. Our new experimental thrombus does not necessarily represent a counterpart of the entire in situ thrombus seen in patients with stroke. However, this method may expand the possibility of an experimental model for preclinical evaluation of mechanical thrombectomy devices.

\section{Conclusions}

We describe a novel method for thrombus preparation that can be used in the evaluation of thrombectomy devices. The experimental thrombus was mechanically stable and histologically similar to the typical thromboemboli recovered from patients with stroke who have undergone thrombectomy. This method may expand the possibility of preclinical evaluation of mechanical thrombectomy devices.

\section{References}

1. Smith WS, Sung G, Saver J, et al. Mechanical thrombectomy for acute ischemic stroke: final results of the Multi MERCI trial. Stroke 2008;39:1205-12. Epub 2008 Feb 28

2. Gralla J, Schroth G, Remonda L, et al. A dedicated animal model for mechanical thrombectomy in acute stroke. AJNR Am J Neuroradiol 2006;27:1357-61

3. Marder VJ, Chute DJ, Starkman S, et al. Analysis of thrombi retrieved from cerebral arteries of patients with acute ischemic stroke. Stroke 2006;37:2086-93

4. Zucker MB, Owen J. Non-decalcified barium sulfate-adsorbed plasma: a potentially useful reagent for studying blood clotting, platelets or complement. Thromb Haemost 1982;47:182-84 